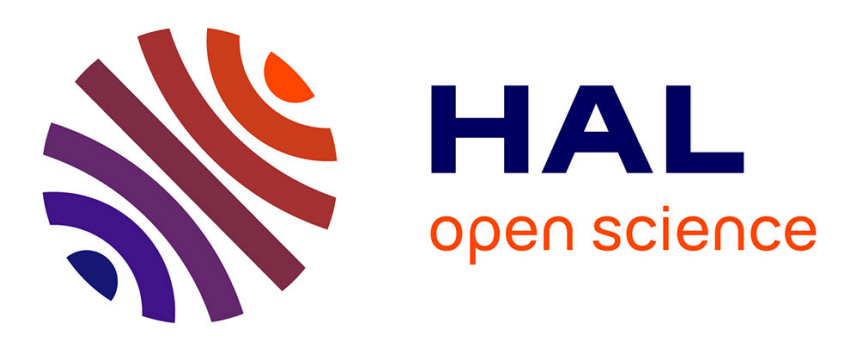

\title{
Description préliminaire d'un potyvirus isolé de courgette (Cucurbita pepo L.) en Guadeloupe
}

\author{
Laurence Quiot-Douine, Jean-Bernard Quiot, Gérard Labonne
}

\section{To cite this version:}

Laurence Quiot-Douine, Jean-Bernard Quiot, Gérard Labonne. Description préliminaire d'un potyvirus isolé de courgette (Cucurbita pepo L.) en Guadeloupe. Agronomie, 1986, 6 (3), pp.227-233. hal-00884870

\section{HAL Id: hal-00884870 \\ https://hal.science/hal-00884870}

Submitted on 1 Jan 1986

HAL is a multi-disciplinary open access archive for the deposit and dissemination of scientific research documents, whether they are published or not. The documents may come from teaching and research institutions in France or abroad, or from public or private research centers.
L'archive ouverte pluridisciplinaire HAL, est destinée au dépôt et à la diffusion de documents scientifiques de niveau recherche, publiés ou non, émanant des établissements d'enseignement et de recherche français ou étrangers, des laboratoires publics ou privés. 


\title{
Description préliminaire d'un potyvirus isolé de courgette (Cucurbita pepo L.) en Guadeloupe
}

\author{
Laurence QUIOT-DOUINE, Jean-Bernard QUIOT \& Gérard LABONNE $\left(^{*}\right)$ \\ Laboratoire de Phytovirologie des Régions chaudes, C.I.R.A.D.-I.N.R.A.-O.R.S.T.O.M., B.P. 5035, F 34032 \\ Montpellier Cedex \\ (*) I.N.R.A., Laboratoire de Recherches de la Chaire de Zoologie de l'Ecole Nationale Supérieure Agronomi- \\ que, F 34060 Montpellier Cedex
} Watermelon Mosaic Virus 1 (WMV-1), est très répandu dans les cultures de courgette où il provoque des symptômes de mosaïque avec cloques et des déformations filiformes très marquées sur le feuillage et également des déformations de fruits.

Depuis 1982, on a constaté qu'un autre faciès maladif était aussi épidémique dans les champs de courgette. II se distingue de celui de PRSV-W par une mosaique donnant un aspect tigré au feuillage et l'absence de déformation filiforme.

L'isolat (Q10) provenant de l'une des plantes infectées par la souche « Tigré » a été étudié. Le virus est transmissible par inoculation mécanique. II est transmis par puceron sur le mode non persistant mais à un taux différent de celui observé pour le PRSV-W. Sa gamme d'hôtes est analogue à celle du PRSV-W. Elle est limitée à la famille des Cucurbitacées et en particulier, les génotypes de Cucumis melo, C. metuliferus, Cucurbita ecuaderensis et $C$. foetidissima résistants au PRSV-W sont aussi résistants à l'isolat Q10.

Les particules virales sont flexueuses. L'infection induit dans le cytoplasme la formation d'inclusions amorphes et d'inclusions cylindriques se classant dans la subdivision $\mathbf{I}$.

Des tests sérologiques d'immunodiffusion en présence de dodécyl-sulfate de sodium et des tests en ISEM (Immunosorbent Electron Microscopy) ont montré que l'isolat Q10 ne réagissait avec aucun des sérums dont nous disposions préparés contre les virus suivants : PRSV-W, Watermelon Mosaic Virus 2 (WMV-2), Watermelon Mosaic Virus "Morocco" (WMV-M), Zucchini Yellow Mosaic Virus (ZYMV), Zucchini Yellow Fleck Virus (ZYFV). Inversement, PRSV-W, WMV2 et ZYMV n'ont pas réagi avec le sérum préparé spécifiquement contre l'isolat Q10.

L'isolat Q10 a des propriétés biologiques proches de celles du PRSV-W; il s'en distingue par son taux de transmission par puceron, sa symptomatologie sur certains hôtes et par ses propriétés sérologiques. Une étude complémentaire permettra d'établir si cet isolat doit être considéré comme un sérotype du PRSV-W ou comme un virus à part entière.

Mots clés additionnels : Virus, virus I de la mosaïque de la pastèque, virus des taches annelées du papayer, watermelon mosaic virus 1, papaya ringspot virus, cucurbitacées, pastèque, caraibes, identification.

Preliminary description of a potyvirus isolated from zucchini squash (Cucurbita pepo L.) in Guadeloupe, a Caribbean island.

In Guadeloupe, a Caribbean island, the watermelon strain of papaya ringspot virus (PRSV-W = watermelon mosaic virus 1 , WMV-1) is commonly encountered in squash fields. It induces a severe mosaic with blistering and filiformism on squash leaves, and a deformation of fruits. Since 1982, a new disease has been found epidemically in squash fields, the symptoms being leaf discoloration according to a striped ("Tigré") pattern and limited filiformism. An isolate (Q10), collected from a squash showing the "Tigré" symptom, was studied. The virus was transmitted mechanically and also transmitted by aphids in the non-persistent manner and less efficiently than PRSV-W. Its host range was similar to that of PRSV-W. It was restricted to the Cucurbit family, and genotypes of Cucumis melo, C. metuliferus, Cucurbita ecuadorensis and C. foetidissima resistant to PRSV-W were also resistant to isolate Q10. Virus particles were filamentous. Infection induced in the cytoplasm the formation of amorphous and cylindrical type 1 inclusions. Sodium dodecyl sulfateimmunodiffusion and ISEM (Immunosorbent Electron Microscopy) tests showed that isolate Q10 did not react with sera prepared against the following viruses: PRSV, watermelon mosaic virus 2 (WMV-2) watermelon mosaic virus "Morocco" (WMV-M), zucchini yellow mosaic virus (ZYMV), zucchini yellow fleck virus (ZYFV). Conversely, PRSV-W, WMV-2 ans ZYMV did not react with the serum specifically prepared against isolate Q10. Some biological properties of isolate Q10 and PRSV-W were similar but the viruses could be distinguished by their aphid transmission rate, symptoms on some hosts and serological properties. Further studies will show whether this isolate should be considered as a new serotype of PRSV or as a new virus.

Additional key words : Virus, watermelon mosaic virus 1, papaya ringspot virus, Cucurbitaceae, watermelon, identification. 


\section{INTRODUCTION}

Parmi les 26 virus reconnus capables d'infecter les Cucurbitacées (I ovisolo, 1980), 2 seulement ont été isolés jusqu'à présent en Guadeloupe. Le premier est le virus de la mosaïque du concombre (CMV) identifié pour la première fois sur bananier en 1966 (YOTDAUTHY \& BOVÉ), le second, le Papaya Ringspot Virus souche WMV-1 (PRSV-W) (ex-Watermelon Mosaic Virus 1, Purcifull et al., 1984a) trouvé dans différentes Cucurbitacées en 1971 (QUIOT et al.).

En 1982, un symptôme particulier (aspect " tigré » du feuillage) est observé dans une parcelle de courgettes cultivées dans le Nord de l'île. Cet article présente la description préliminaire des propriétés biologiques d'un isolat produisant le symptôme «tigré » sur courgette. Des études sur les relations sérologiques de cet isolat avec d'autres virus des Cucurbitacées sont présentées dans un article à paraître (QUIOT-DOUINE et al., 1986).

\section{MATÉRIEL ET MÉTHODES}

L’isolat «Tigré » Q10 avec lequel la plupart des études ont été réalisées a été collecté en mars 1982 sur une courgette (Cucurbita pepo L. var. "Diamant F1 》) naturellement infectée ; d'autres isolats "Tigré » proviennent soit d'autres plantes de la même culture, soit de cultures ultérieures réalisées sur le même emplacement ou dans son voisinage.

Le virus transmis à des courgettes par inoculation mécanique selon la méthode décrite par MARROU (1967) est conservé sur plantes en serre grillagée étanche aux insectes ou dans des feuilles desséchées sur chlorure de calcium à $+4{ }^{\circ} \mathrm{C}$.

La recherche des espèces sensibles à l'isolat Q10 se fait par inoculation mécanique de une ou plusieurs plantes de chaque espèce en un ou plusieurs tests. Toutes les plantes qui n'ont pas présenté de symptômes et certaines plantes ayant réagi positivement sont contrôlées par rétroinoculation mécanique sur courgettes à partir des feuilles apicales. Dans le cas du pois (Pisum sativum L. var. "Express Alaska »), les feuilles inoculées sont également contrôlées (PURCIFULL $e t$ al., 1984b).

Les transmissions par pucerons sont réalisées avec Aphis gossypii Glov. à partir de courgettes « Diamant $\mathrm{F} 1$ » infectées depuis $14 \mathrm{j}$. Les adultes aptères sont mis à jeûner pendant $1 \mathrm{~h} 30$; l'acquisition dure $3 \mathrm{mn}$ et la transmission à de jeunes courgettes « Diamant F1 » a lieu immédiatement après l'acquisition à raison de 2 pucerons par plante.

Les observations en microscopie optique sont réalisées sur des lambeaux d'épiderme de courgette «Diamant F1 " traités par une solution à 5 p. 100 de triton X100 puis colorés avec le mélange calcomine orangevert brillant (OG) selon la technique décrite par CHRISTIE \& EDWARDSON (1977).

Les observations en microscopie électronique sont réalisées sur coupes ultraminces de limbes de courgettes «Diamant $F 1$ » infectées depuis $10 \mathrm{j}$, incluses dans l'araldite et doublement colorées avec 5 p. 100 d'acétate d'uranyle et une solution de citrate de plomb à $\mathrm{pH} 12$.
La purification du virus est réalisée à partir de courgettes var. "Diamant F1 " infectées depuis 15 à 25 i en utilisant une technique adaptée de celle décrite par PURCIFUlL \& HIEBERT (1979) pour la souche Watermelon Mosaic Virus 1 du PRSV.

Après chaque purification, le pouvoir infectieux du produit final est contrôlé par inoculation d'une série de dilutions à des courgettes (3 par dilution).

Un sérum spécifique de la protéine capside du virus a été obtenu par injections à un lapin de préparations virales préalablement traitées avec 3 p. 100 de sodium dodécyl sulfate (SDS) et 3 p. 100 de 2-mercaptoéthanol et chauffées $2 \mathrm{mn}$ dans un bain-marie bouillant.

Les tests sérologiques sont réalisés selon la technique d'immunodiffusion en milieu gélosé en présence de SDS décrite par PURCIIULL \& BATCHELOR (1977) ou selon la méthode ISEM (Immunosorbent Electron Microscopy) mise au point par DERRICK \& BRANLSKY (1976).

Les autres sérums anti-protéine capside utilisés sont : le sérum $n^{\circ} 643$ anti PRSV-W originaire de Floride (D. PURCIFULL, non publié), de Guadeloupe (H. LOT, non publié) ou d'Italie (Russo et al., 1979), le sérum $n^{\circ} 955$ anti Watermelon Mosaic Virus originaire du Maroc (WMV-M) (BAUM et al., 1979), le sérum $\mathrm{n}^{\circ} 868$ anti Watermelon Mosaic Virus 2 (WMV-2) originaire de Floride (PURCIFUlL \& HIEBERT, 1979) ou de France (H. LECOQ, non publié), le sérum anti Zucchini Yellow Mosaic Virus (ZYMV) originaire de France (LECOQ et al., 1981, 1983) ou d'Italie (LISA et al., 1981 ; LECOQ et al., 1983) et le sérum anti Zucchini Yellow Fleck Virus (ZYFV) originaire d'Italie (VovLAs et al., 1981).

\section{RÉSULTATS}

\section{A. Symptômes sur courgette var. «Diamant F1"}

Au champ, les courgettes atteintes se distinguent par l'aspect terne et gaufré de leurs feuilles. Celles-ci présentent également des taches peu contrastées, d'un vert plus clair, induisant parfois un léger renforcement de coloration le long des nervures (vein banding), mais ne subissent pas de déformation très filiforme. Les fruits sont très déformés, parfois fortement courbés, avec des creux très accentués et, dans certains cas, des variations de diamètre (fig. 1).

En serre, la courgette var. "Diamant F1" inoculée mécaniquement avec l'isolat Q10 montre sur les feuilles des bandes de décoloration plus ou moins forte partant du bord du limbe et allant en se rétrécissant vers le centre. Les feuilles peuvent parfois présenter une légère crispation au niveau de la décoloration et, quelquefois, un rétrécissement du limbe mais sans jamais atteindre un aspect filiforme comme dans le cas de la souche W du PRSV (fig. 2).

\section{B. Observations sur l'épidémiologie du virus}

La zone dans laquelle le virus a été trouvé était en observation depuis 1979: 3 cultures successives de courgettes y étaient plantées chaque année et les plantes étaient régulièrement testées par inoculation méca- 


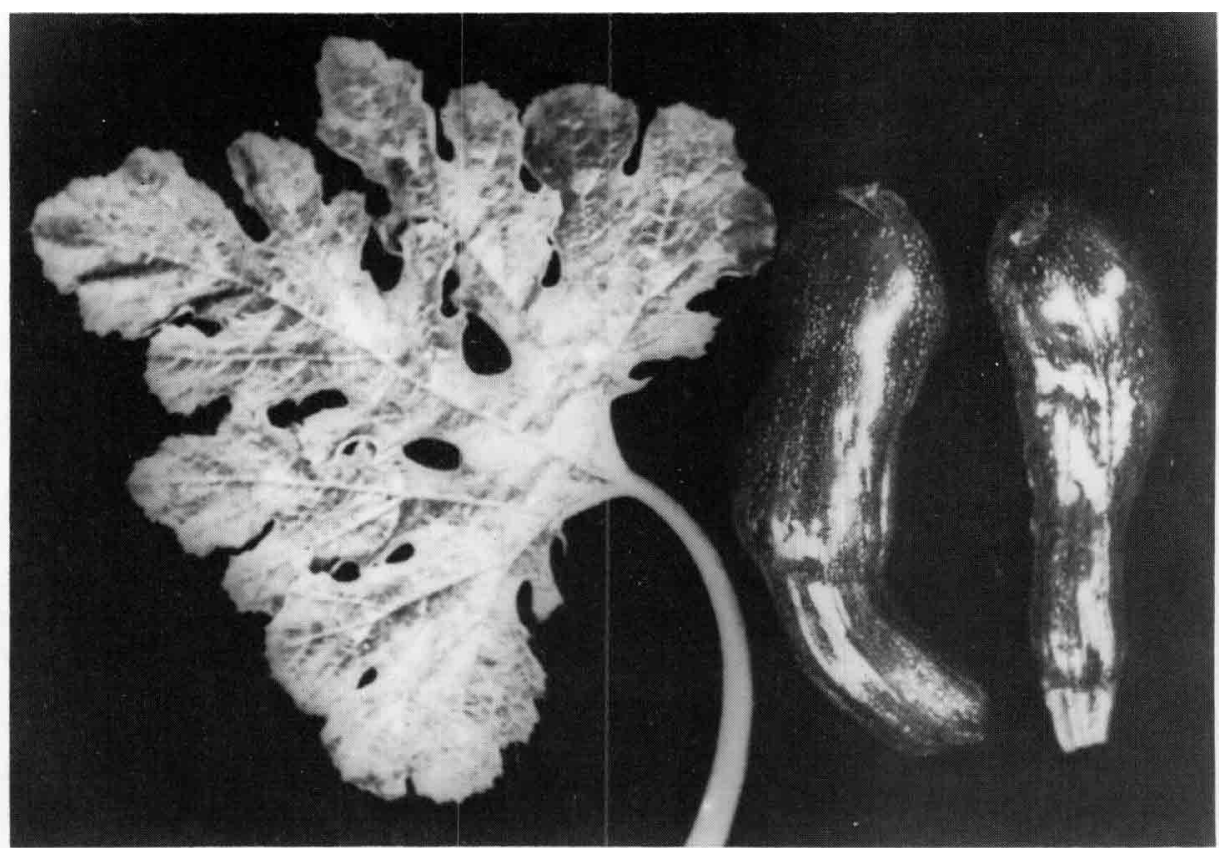

Figure 1

Infection naturelle au champ, de courgette var. "Diamant FI" par l'isolat QIO. Symptômes sur feuille et sur' fruit.
Natural field infection of zucchini squash var. "Diamant Fl 》 by isolate QIO. Symptoms on leaf and fruit.

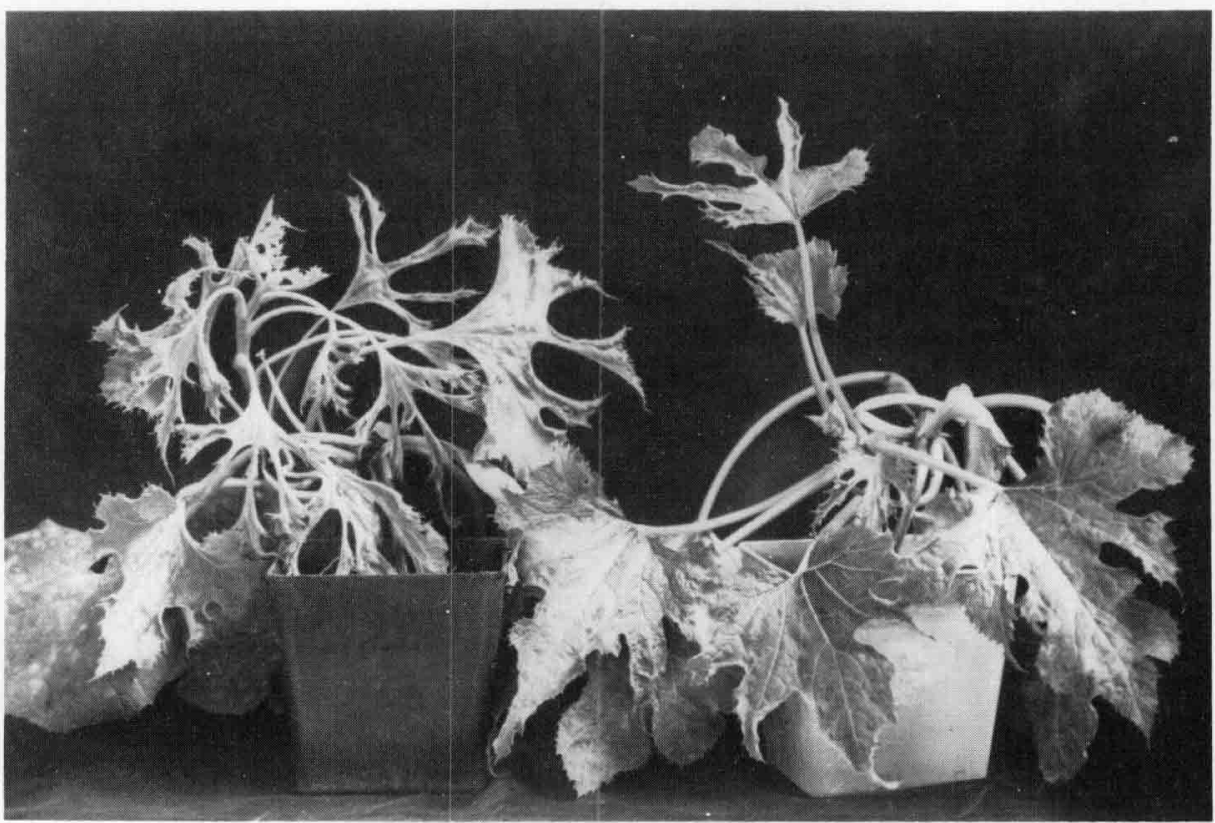

Figure 2

Comparaison des symptômes produits en serre sur courgette « Diamant FI " après inoculation mécanique par: à gauche, la souche Watermelon Mosaic Virus 1 (WMV-l) de Papaya Ringspot Virus (PRSV-W) originaire de Guadeloupe et à droite, l'isolat Q10.
Comparison of symploms produced in the greenhouse on zucchini squash var. «Diamant FI», mechanically inoculated with the watermelon mosaic virus (WMV-I) strain of papaya ringspot virus (PRSV-W) from Guadeloupe on left, and isolate QIO on right. nique à une gamme d'hôtes restreinte et par sérologie pour y rechercher le CMV et le PRSV-W. Ce n'est qu'au printemps 1982 que l'on a commencé à trouver des plantes qui présentaient des symptômes transmissibles par inoculation mécanique mais donnaient des réponses négatives en sérologie, révélant ainsi l'existence d'un $3^{\mathrm{e}}$ virus. Ce virus a été retrouvé par la suite dans 3 essais successifs répartis sur plus d'une année. Il semble cependant avoir une dissémination plus lente que le PRSV-W. En effet, dans certains essais, alors que des infections naturelles simultanées par PRSV-W et par la souche «Tigré » avaient été précocement détectées, la souche "Tigré » était, par la suite, supplantée dans la parcelle par le PRSV-W. 
Plusieurs plantes ont été trouvées infectées par les 2 virus à la fois mettant en évidence la possibilité d'infection mixte par le PRSV-W et le nouveau virus. Des inoculations mécaniques réalisées au laboratoire ont confirmé que les 2 virus pouvaient infecter simultanément la même plante.

\section{Gamme d'hôtes}

On constate que la gamme d'hôtes sensibles à l'isolat Q10 est limitée aux Cucurbitacées (tabl. 1).

TABLEAU 1

Gamme d'hôtes comparative de l'isolat QIO et de la souche Watermelon Mosaic Virus 1 (WMV-I) du Papaya Ringspot Virus (PRSV-W).

Comparative host range of isolate $Q 10$ and watermelon mosaic virus $1(W M V-1)$ strain of papaya ringspot virus (PRSV-W).

\begin{tabular}{|c|c|c|c|c|}
\hline \multirow[b]{2}{*}{ Hôtes } & \multicolumn{2}{|c|}{ Isolăt Q10 } & \multicolumn{2}{|c|}{ PRSV $-W(*)$} \\
\hline & Sympt. & $\begin{array}{l}\text { Rétro- } \\
\text { inoc. }\end{array}$ & Sympt. & $\begin{array}{l}\text { Rétro- } \\
\text { inoc. }\end{array}$ \\
\hline $\begin{array}{l}\text { Citrullus vulgaris Schrad. } \\
\text { var. « Sugar Baby» }\end{array}$ & $\frac{0}{(\mathrm{Mo})}$ & + & - & - \\
\hline Cucumis anguria $\mathrm{L}$. & $\frac{0}{(\mathrm{Mo})}$ & + & 0 & + \\
\hline $\begin{array}{l}\text { Cucumis melo } \mathrm{L} \text {. } \\
\text { var. « Chilton» }\end{array}$ & $\frac{\mathrm{Sp}}{\mathrm{Sp}} \frac{\mathrm{CN}, \mathrm{N}}{\mathrm{CN}, \mathrm{N}}$ & + & $\frac{\text { Sp CN, N }}{\text { Sp CN, N }}$ & + \\
\hline var. «Doublon" & $\frac{\mathrm{SpCN}, N}{\operatorname{SpCN}, N}$ & + & $\frac{0}{\mathrm{VC}}$ & - \\
\hline var. "Védrantais » & $\frac{\operatorname{SpCN}, N}{\operatorname{SpCN}, N}$ & + & $\frac{0}{V C}$ & - \\
\hline PI 161375 & 0 & 0 & 0 & 0 \\
\hline WMR 29 (PI 180280) & 0 & 0 & - & - \\
\hline $72025 \quad$ (PI 180283) & 0 & 0 & - & - \\
\hline D9A $\quad$ (PI 414723) & 0 & 0 & - & - \\
\hline Cucumis metuliferus MCY. & 0 & 0 & - & - \\
\hline $\begin{array}{l}\text { Cucumis sativus L. } \\
\text { var. "Gemini " }\end{array}$ & $\frac{(\mathrm{N})}{(\mathrm{N})}$ & + & $\frac{0}{\mathrm{Mo}}$ & - \\
\hline $\begin{array}{l}\text { var. Verı Long Maraîcher } \\
\text { Cucurbita ecuadorensis }\end{array}$ & 0 & + & $\frac{0}{(\mathrm{Mo})}$ & - \\
\hline Cutl. \& Whit. & () & - & - & - \\
\hline Cucurbira foetidissima $\mathrm{HBK}$. & 0 & - & - & - \\
\hline Cucurbita martinezii Bailey & $\frac{0}{M o}$ & - & - & - \\
\hline Cucurbita maxima Duch. & $\frac{0}{\mathrm{Mo}}$ & - & - & - \\
\hline $\begin{array}{l}\text { Cucurbita moschata Duch. } \\
\text { origine Anguilla }\end{array}$ & $\frac{0}{\mathrm{Mo}}$ & - & - & - \\
\hline $\begin{array}{l}\text { Cucurbita pepo L. } \\
\text { var. "Black Jack» }\end{array}$ & $\frac{0}{\text { Mo, D }}$ & + & $\frac{0}{\mathrm{Mo}, \mathrm{Cl}, \mathrm{Fil}}$ & + \\
\hline var. "Chefini" & $\frac{0}{\mathrm{No}, \overline{\mathrm{D}}}$ & + & $\frac{0}{\text { Mo,Cl,Fil }}$ & + \\
\hline var. « Diamant F1» & $\frac{0}{\mathrm{Mo}, \mathrm{D}}$ & + & $\frac{0}{\text { Mo,Cl,Fil }}$ & + \\
\hline $\begin{array}{l}\text { Ecballium elaterum } \\
\text { A. Rich. }\end{array}$ & 0 & 0 & 0 & 0 \\
\hline Luffa acutangula Roxb. & 0 & 0 & 0 & 0 \\
\hline Momordica balsamina $\mathrm{L}$ & $\frac{0}{(\mathrm{Mo})}$ & + & - & - \\
\hline $\begin{array}{l}\text { Sechium edule Schwartz } \\
\text { Chenopodium amarantico- }\end{array}$ & 0 & 0 & 0 & 0 \\
\hline lor Coste \& Reyn. & 0 & 0 & 0 & 0 \\
\hline Chenopodium quinoa Willd & 0 & 0 & $\begin{array}{l}0 \\
0\end{array}$ & 0 \\
\hline $\begin{array}{l}\text { Lavatera trimestris } \mathrm{L} \text {. } \\
\text { Nicotiana benthamiana } \\
\text { Domin. }\end{array}$ & $\begin{array}{l}0 \\
0\end{array}$ & $\begin{array}{l}0 \\
0\end{array}$ & $\begin{array}{l}0 \\
0\end{array}$ & $\overrightarrow{0}$ \\
\hline $\begin{array}{l}\text { Pisum sativum L. } \\
\text { var. «Express Alaska » }\end{array}$ & 0 & $\begin{array}{l}\text { f. inoc. } 0 \\
\text { f. syst. } 0\end{array}$ & 0 & $\begin{array}{l}\text { f. inoc. } 0 \\
\text { f. syst. } 0\end{array}$ \\
\hline
\end{tabular}

Il est à noter que Luffa acutangula Roxb., bien que trouvé hôte du PRSV-W par de nombreux chercheurs (WEBB \& SCOTT, 1965; PROVVIDENTI \& SCHROEDER, 1970; Greber, 1978; PURCIFUll \& HIEBERT, 1979; RUSSO et al., 1979), n'a pu être infecté de façon systémique dans nos conditions, au cours de 3 essais différents, ni par l'isolat Q10 ni par 2 souches de PRSV-W originaires, l'une de Guadeloupe, l'autre de Floride (WMV-1 Fl., PurCIFUll \& HIEBERT, 1979) inoculées parallèlement. Toutefois l'isolat Q10 a pu être détecté par sérologie dans les feuilles inoculées.

Le papayer a été plus particulièrement étudié, dans l'hypothèse où l'isolat Q10 serait un isolat de PRSVP. Au cours de 6 essais différents, 27 plants de papayers var. "Solo", dont le stade variait de 1 à 13 feuilles, ont été inoculés avec des extraits dont le pouvoir infectieux était vérifié par inoculation simultanée à des courgettes. Un seul papayer a présenté des symptômes de mosaïque. Tous les autres, non seulement ne présentaient pas de symptômes, mais sc révélaient, par rétroinoculation, indemnes de virus. $\mathrm{La}$ rétroinoculation à partir du papayer malade, n'a permis d'infecter qu'une seule des 2 courgettes inoculées. Un test sérologique a permis de vérifier que cette courgette contenait le sérotype isolat Q10.

Les espèces suivantes appartenant à différentes familles n'ont pas été trouvées sensibles à l'isolat Q10 : Solanacées : Capsicum annuum L. var. "YoloWonder ", Datura stramonium L., Lycopersicon esculentum Mill. var. " Floradel », Nicotiana benthamiana Domin., N. glutinosa L., N. tabacum L. var. "Xanthi nc." et Solanum melongena L. var. "Violette de Barbentane »; Fabacées : Gliricidia sepium Kunth ex Walp., Leucaena leucocephala (Lam.) de Wit. Laedtree, Phaseolus lunatus L., P. vulgaris L. var. "Bountifull", var. "Contender" et var. «Pinto », Pisum sativum L. var. « Express Alaska» et var. "Douce Provence ", Vicia faba L. var. "Superaguadulce », Vigna radiata (L.) Wilczek et $V$. unguiculata $(\mathrm{L}$.$) Walp. (=V$. sinensis $(\mathrm{L}$.$) Endl.) var.$ «Black» et parmi d'autres familles : Apium graveolens L. var. "Doré Barbier ", Brassica napus (L.) Metzger, Chenopodium amaranticolor Coste et Reyn.,

\begin{tabular}{ll}
\hline symptôme local & local symptom \\
symptôme systémique & systemic symptoms \\
$+\quad$ résultat positif ; & positive result \\
$0 \quad$ pas de symptôme ; & no symptoms \\
$-\quad$ non testć ; & not tested \\
( ) symptôme erratique ; & crratic symptoms \\
$\mathrm{C}$ chlorotique ; & chlorotic \\
$\mathrm{Cl}$ cloque ; & blister \\
Fil filiforme ; & filiform \\
Mo mosaïque ; & mosaic \\
$\mathrm{N}$ nécrose ; & necrosis \\
VC éclaircissement des nervures ; & vein clearing
\end{tabular}

(*) Les résultats donnés pour la souche WMV-1 du PRSV ne concernent que les essais réalisés en parallèle entre l'isolat Q10 et la souche originaire de Guadeloupe.

Results given here for the WMV-1 strain of PRSV are only those obtained with a Guadeloupe strain inoculated at the same time as isolate Q10. 
C. quinoa Willd., Cyamopsis tetragonoloba (L.) Taub., Gomphrena globosa L., Lactuca sativa L. var. "Trocadéro ", Lavatera trimestris L., Melochia pyramidata L., Ocimum basilicum L., Zinnia elegans Jacq. et Zisiphus mauritiana Lam.

\section{Transmission par aphide}

Des essais de transmission par puceron réalisés au laboratoire ont montré que l'isolat Q10 était transmis sur le mode non persistant. Dans les conditions décrites précédemment, 3 plantes sur les 20 testées ont été infectées par l'isolat Q10, alors que dans des conditions similaires, 17 plantes sur les 30 inoculées ont été infectées par l'isolat guadeloupéen du PRSV'-W. La différence du taux de transmission par puceron de ces 2 virus est significative $\left(\chi^{2}=7,03\right)$.

\section{E. Observations au microscope}

Au microscope électronique, le virus apparaît sous forme de particules flexueuses, relativement peu nombreuses dans les extraits de plantes incubées à $25^{\circ} \mathrm{C}$.

Il induit dans le cytoplasme 2 types d'inclusions observables au microscope optique après coloration de lambeaux de feuilles avec le mélange OG (fig. 3). Les inclusions cylindriques de type " pinwheel » apparaissent sous forme de fagots colorés en brun-vert selon les temps de coloration. Les inclusions amorphes ou inclusions cytoplasmiques irrégulières se présentent comme des amas plus foncés de forme plus ou moins arrondie.

L'observation au microscope électronique révèle la présence d'inclusions cylindriques ou "pinwheels" associées à des rouleaux uniquement (fig. 4).

\section{F. Purification}

Des pré-essais ont montré que le virus résiste à un traitement par le tétrachlorure de carbone à 15 et à 25 p. 100 , le chloroforme à 33 p. 100 ou un mélange de 25 p. 100 de chloroforme et 25 p. 100 de tétrachlorure de carbone. Par contre, son pouvoir infectieux est totalement détruit par 8 p. 100 de $n$-butanol.

Des pertes importantes ont lieu au cours des clarifications à basse vitesse $(3000 \mathrm{~g}$ pendant $10 \mathrm{mn}$ ) et le virus précipité est encore infectieux.

Le virus présente une absorption maximum à $258 \mathrm{~nm}$ et une absorption minimum à $240 \mathrm{~nm}$. Les 5 meilleures purifications, jugées à la fois par le spectre de la préparation finale de virus et par son pouvoir infectieux, montrent que le rapport DO max./DO min est de $1,32 \pm 0,15$, le rapport DO 260/DO 280 de $1,43 \pm 0,28$ et le rapport DO $260 / \mathrm{DO} 240$ de $1,20 \pm 0,26$.

La suspension de virus purifié est encore infectieuse à une dilution représentant le $1 / 25^{\mathrm{e}} \mathrm{du}$ poids de feuilles de départ mais ne l'est plus au 1/625e. Par comparaison, dans les mêmes conditions, des courgettes peuvent encore être infectées, à partir d'extrait brut, à une dilution égale au $1 / 2500^{\mathrm{e}}$ mais non à la dilution suivante $\left(1 / 12500^{\mathrm{e}}\right)$.

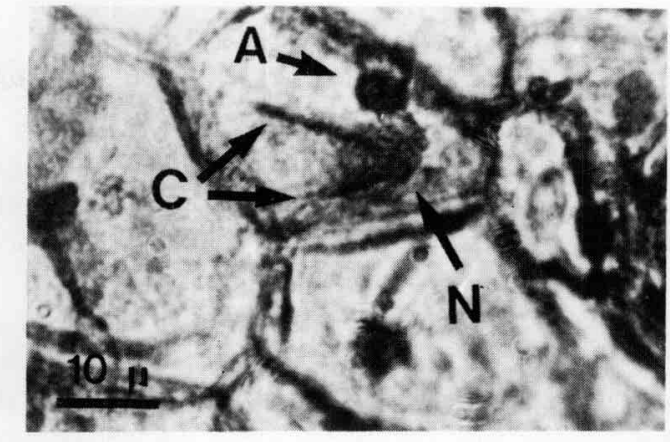

Figure 3

Inclusions cytoplasmiques observées au microscope optique dans des cellules de courgette infectée par l'isolat Q10, après traitement par triton X 100 à $5 \mathrm{p} .100$ et coloration par le mélange calcomine orange-vert brillant (OG) $(\times 1024)$.

Cytoplasmic inclusions seen by light microscopy in cells of zucchini squash infected bv isolate 010 , treated with $5 \%$ triton $X 100$ and stained with the calcomine orange-luxol brilliant green combination $(O G)(\times 1,024)$

$A=$ inclusion amorphe ; amorphous inclusion.

$C=$ inclusions cylindriques $;$ cylindrical inclusions.

$N=$ noyau; nucleus.

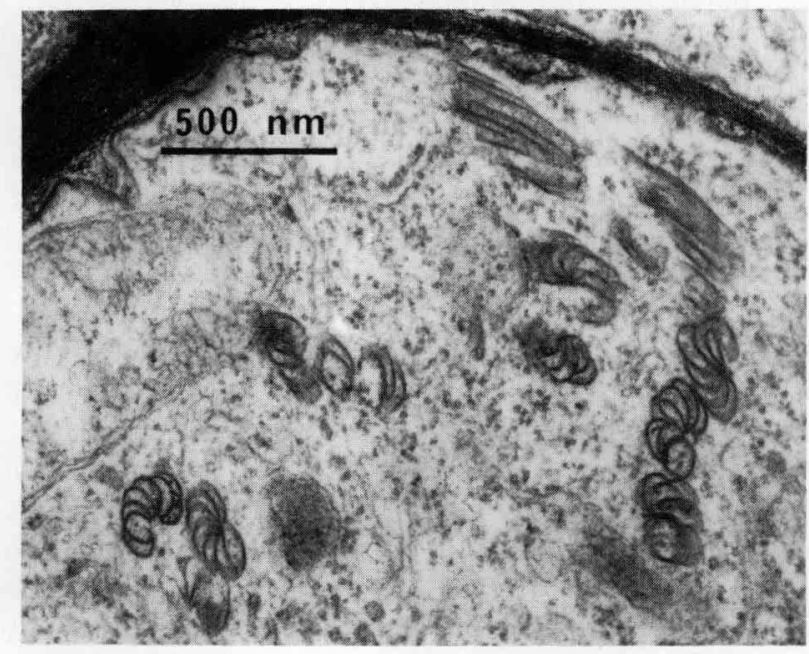

Figure 4

Coupe ultramince de feuille de courgette infectée par l'isolat $Q 10$, montrant des inclusions cylindriques en rouleaux $(\times 36500)$.

Ultrathin section of zucchini squash leaf infected with isolate Q10, showing scrolls $(\times 36,500)$.

\section{G. Sérologie}

\section{1) Préparation d'un antisérum}

Un antisérum est obtenu à partir d'un lapin ayant reçu 5 injections intramusculaires de $3 \mathrm{mg}$ de virus espacées de 21 à $27 \mathrm{j}$. Le sérum prélevé $28 \mathrm{j}$ après la $1^{\text {re }}$ piqûre ne réagit pas avec le virus. Une prise de sang faite 15 semaines après la $1^{\text {re }}$ piqûre et une autre 16 semaines après, mais précédée $8 \mathrm{j}$ avant d'une piqûre de rappel, fournit du sérum réagissant avec le virus mais non avec les extraits de plante saine. En immunodiffusion, le sérum réagit encore à la dilution $1 / 4$. 


\section{2) Tests sérologiques}

En immunodiffusion-SDS, le virus n'a réagi avec aucun des antisérums préparés contre les virus suivants : PRSV-W qu'il soit originaire de Guadeloupe ou de Floride (fig. 5) ; WMV-M ; WMV-2 originaire de Floride ou de France ; ZYMV originaire de Floride ou de France.

Inversement, aucun des virus suivants ne réagit avec le sérum préparé contre l'isolat Q10 : PRSV-W originaire de Guadeloupe ou de Floride (fig. 5) ; WMV-2 originaire de Floride ou de France ; ZYMV originaire de France.

La technique ISEM confirme que l'isolat Q10 ne réagit pas avec les sérums anti-protéine capside du PRSV-W de Guadeloupe, du WMV-2 de France et du ZYMV, qu'il soit originaire de France ou d'Italie. Cette technique montre qu'il n'y a pas de réaction entre le virus et des sérums anti-protéine capside de PRSV-W, de ZYMV et de ZYFV, tous 3 originaires d'Italie.

\section{CONCLUSION}

Par sa forme flexueuse, son mode de transmission non persistant par aphide et les inclusions de type "pinwheel " qu'il induit à l'intérieur des cellules infectées, l'isolat Q10 se révèle appartenir au groupe des potyvirus.

Tous les hôtes de l'isolat Q10 appartiennent à la famille des Cucurbitacées. Certains de ces hôtes sont communs avec d'autres potyvirus. Cependant, l'absence de réaction à l'isolat Q10 d'hôtes différentiels tels que $C$. amaranticolor et $C$. quinoa, $L$. trimestris, $N$. benthamiana et $P$. sativum var. "Express Alaska " permet de le différencier des WMV-2 et WMV-M (WEBB \& SCOTT, 1965; EDWARDSON, 1974 ; FISCHER \& LOCKHART, 1974 ; PURCIFULL \& HIEBERT, 1979), du ZYMV (LISA et al., 1981 ; LECOQ et al., 1981, 1983), du Bean Yellow Mosaic Virus (BYMV) (PROVVIDENTI \& UYEMOTO, 1973 ; KOMM \& Agrios, 1978 ; Lisa \& Dellavali.e, 1981) et du Bryonia Mottle Virus (LoCKHART \& FISCHER, 1979).

Les propriétés sérologiques de l'isolat Q10 confirment qu'il est différent de ces virus et également du ZYFV. Toutefois, il convient de préciser que les sérums utilisés sont très spécifiques de leur virus homologue respectif.

L'isolat Q10 se différencie aussi du WMV-2 par le type d'inclusions cylindriques qu'il induit dans le cytoplasme de l'hôte. Ces inclusions permettent de le classer dans la subdivision I, comme les PRSV-P et W (EDWARDSON, 1974).

L'isolat Q10 se distingue du PRSV-P par un taux d'infection des papayers extrêmement faible et par les nécroses qu'il induit sur melon alors que, selon la littérature, le PRSV-P ne semble pas infecter le melon (ZETTLER et al., 1968 ; STORY \& HALliWell, 1969).

L'isolat Q10 présente certaines différences avec le PRSV-W : quelques variations au niveau de l'expression du type de symptôme sur certains hôtes et, pour un même hôte, un taux plus faible de transmission par puceron se traduisant par une capacité de dissémination au champ plus limitée. Par contre, il possède des propriétés physiques et biologiques importantes très
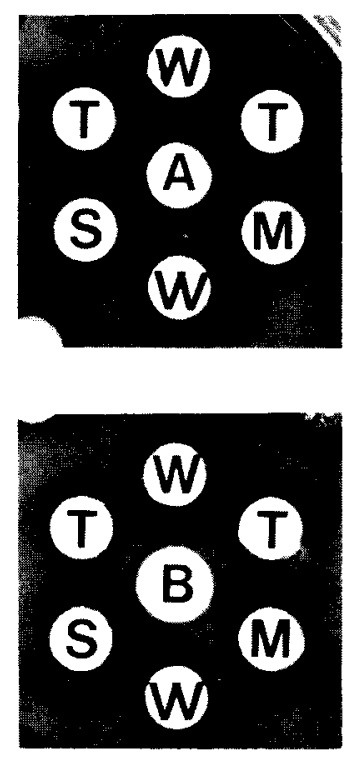

Figure 5

Réactions sérologiques en immunodiffusion en présence de dodécyl sulfale de sodium (SDS). Les puils centraux contenaient les sérums non dilués $n^{\circ} 643$ anti-souche Watermelon Mosaic Virus 1 (WMVl) dir Papava Ringspot Virus (PRSV) (A) et $n^{\circ}$ 83-IC anti-isolat $Q I 0(B)$. Les puits périphériques contenaient les antigènes dans des extraits bruts (dilués au $1 / 3$ et traités avec $1 \mathrm{p}$. 100 de SDS) de feuilles de courgettes infectées avec : l'isolat $Q I 0(T)$, le PRSV-W (W), et le Watermelon Mosaic Virus originaire du Maroc (WMV-M) (M) oul saines $(S)$.

Serological reacions in sodium dodecyl sulfate (SDS)immunodiffusion. Center wells contained undiluted antisera : $n^{\circ}$ 643 to the watermelon mosaic virus I (WMV-l) strain of papaya ringspot virus (PRSV-W) $(A)$ and $n^{\circ} 83-l C$ to isolate $Q I O(B)$. Peripheral wells contained I\% SDS-treated antigens in crude extracts (dilution 1/3) of zucchini squash leaves infecred with : isolate Q10 (T), PRSV $W$ (W), and watermelon mosaic virus from Morocco (WMV-M) (M) or healthy (S).

proches de celles de PRSV-W : l'isolat Q10 est inactivé par le $n$-butanol comme l'est le PRSV-W (HIEBERT, in PURCIFUll et al., 1984a) et sa gamme d'hôtes est identique à celle du PRSV-W. En particulier, les génotypes de Cucumis melo L., C. metuliferus MCY., Cucurbita foetidissima Hвк. et C. ecuadorensis Cutl. \& Whit. qui sont résistants au PRSV-W sont aussi résistants à l'isolat Q10.

Par ailleurs, l'apparition soudaine de ce virus dans une zone cultivée depuis 3 ans avec des courgettes rapidement infectées à un fort pourcentage par le PRSV-W, renforce l'hypothèse que la souche «Tigré » pourrait être le résultat de mutations du PRSV-W. Ce mutant est viable en conditions naturelles puisqu'il a été retrouvé dans 3 cultures successives de courgettes étalées sur 13 mois. Cependant, on n'observe pas de protection croisée entre le PRSV-W et l'isolat "Tigré ". De plus, le sérum anti PRSV-W utilisé, comme le sérum anti isolat $Q 10$ préparé, n'ont pas présenté de réaction avec le virus hétérologue. Des études plus approfondies permettront d'établir les liens de parenté sérologique existant entre l'isolat Q10 et le PRSV-W et de déterminer s'il faut considérer l'isolat Q10 comme un sérotype nouveau du PRSV-W ou comme un nouveau virus à part entière. 


\section{REMERCIEMENTS}

Nous tenons à remercier R. BAUM, H. LECOQ, V. LISA, H. LOT, D. Purcifuli, M. Russo \& C. Vovias pour les sérums qu'ils nous ont fournis, ainsi que B. Delecolte pour son aide lors des études en microscopie électronique.

\section{RÉFÉRENCES BIBLIOGRAPHIQUES}

Baum R. H., Purcifull D. E., Hiebert E., 1979. Purification and serology of a Moroccan isolate of Watermelon Mosaic Virus (WMV) (Abstr.). Phytopathology, 69, 1021-1022.

Christie R. G., Edwardson J. R., 1977. Light and electron microscopy of plant virus inclusions. Fla. Agric. Exp. Stn. Monogr. Ser., $9,150 \mathrm{p}$.

Derrick K. S., Branlsky R. H., 1976. Assay for viruses and mycoplasmas using serologically specific electron microscopy. Phytopathology, 66, 815-820.

Edwardson J. R., 1974. Some properties of the Potato Virus Y-group. Fla. Agric. Exp. Sin. Monogr. Ser., 4, 398 p.

Fïscher H. U., Lockhart B. E. L., 1974. Serious losses in cucurbits caused by watermelon mosaic virus in Morocco. Plant Dis. Rep., 58, 143-146.

Greber R.S., 1979. Watermelon mosaic virus 1 and 2 in Queensland cucurbit crops. Aust. J. Agric. Res., 29, 1235-1245.

Komm D. A., Agrios G. N., 1978. Incidence and epidemiology of viruses infecting cucurbit crops in Massachusetts. Plant Dis. Rep., 62, 746-750.

Lecoq H., Pitrat M., Clément M., 1981. Identification et caractérisation d'un potyvirus provoquant la maladie du rabougrissement jaune du melon. Agronomie, 1, 827-834.

Lecoq H., Lisa V., Dellavalle G., 1983. Serological identity of muskmelon yellow stunt and zucchini yellow mosaic viruses. Plant Dis, , 67, 824-825.

Lisa V., Dellavalle G., 1981. Characterization of two potyviruses in Cucurbita pepo. Phytopathol. Z., 100, 279-286.

Lisa V., Boccardo G., D'Agostino G., Dellavalle G., D'Aquilo M., 1981. Characterization of a potyvirus that causes zucchini yellow mosaic. Phytopathology, 71, 667-672.

Lockhart B. E. L., Fischer H. U., 1979. Host range and some properties of Bryonia mottle virus, a new member of the potyvirus group. Phytopathol. Z., 96, 244-250.

Lovisolo O., 1980. Virus and viroid diseases of cucurbits. Acta Hortic., 88, 33-82.

Marrou J., 1967. Amélioration des méthodes de transmission mécanique des virus par absorption des inhibiteurs d'infection sur le charbon végétal. C.R. Acad. Agric. Fr., 53, 972-981.

Provvidenti R., Schroeder W. T., 1970. Epiphytotic of watermelon mosaic among Cucurbitaceae in central New York in 1969. Plant Dis. Rep., 54, 744-748.
Provvidenti R., Uyemoto J. K., 1973. Chlorotic leaf spotting of yellow summer squash caused by the severe strain of bean yellow mosaic virus. Plant Dis. Rep., 57, 280-282.

Purcifull D. E., Batchelor D. L., 1977. Immunodiffusion tests with sodium dodecyl sulfate (SDS)-treated plant viruses and plant viral inclusions. Fla. Agric. Exp. Stn. Tech. Bull., 788, 39 p.

Purcifull D. E., Hiebert E., 1979. Serological distinction of watermelon mosaic virus isolates. Phytopathology, 69, 112-116.

Purcifull D. E., Edwardson J. R., Hiebert E., Gonsalves D., $1984 a$. Papaya ringspot virus. CMI/AAB Descr. Plant Viruses $n^{\circ} 292$ (revised).

Purcifull D. E., Adlerz W. C., Simone G. W., Hiebert E., Christie R. G., 1984b. Serological relationships and partial characterization of zucchini yellow mosaic virus isolated from squash in Florida. Plant Dis., 68, 230-233.

Quiot J. B., Kaan F., Beramis M., 1971. Identification d'une souche de la mosaïque de la pastèque (Watermelon Mosaic Virus 1) aux Antilles françaises. Ann. Phytopathol., 3, 127-132.

Quiot-Douine L., Purcifull D. E., Hiebert E., De Mejia M. V. G., 1986. Serological relationships and in vitro translation of an antigenically distinct strain of papaya ringspot virus (soumis à Phytopathology)

Russo M., Martelli G. P., Vovlas C., Ragozzino A., 1979. Comparative studies on Mediterranean isolates of watermelon mosaic virus. Phytopathol. mediterr., 18, 94-101.

Story G. E., Halliwell R. S., 1969. Identification of distortion ringspot virus disease of papaya in the Dominican Republic. Plant Dis. Rep., 53, 757-760.

Vovlas C., Hiebert E., Russo M., 1981. Zucchini yellow fleck virus a new potyvirus of zucchini squash. Phytopathol. mediterr., 20, 123-128.

Webb R. E., Scott H. A., 1965. Isolation and identification of watermelon mosaic viruses 1 and 2. Phytopathology, 55, 895-900.

Yot-Dauthy D., Bové J. M., 1966. Mosaique du bananier, identification et purification de diverses souches du virus. Bull. Soc. Physiol. Vég., 12, 380-390.

Zettler F. W., Edwardson J. R., Purcifull D. E., 1968. Ultramicroscopic differences in inclusions of papaya mosaic virus and papaya ringspot virus correlated with differential aphid transmission. Phytopathology, 58, 332-335. 